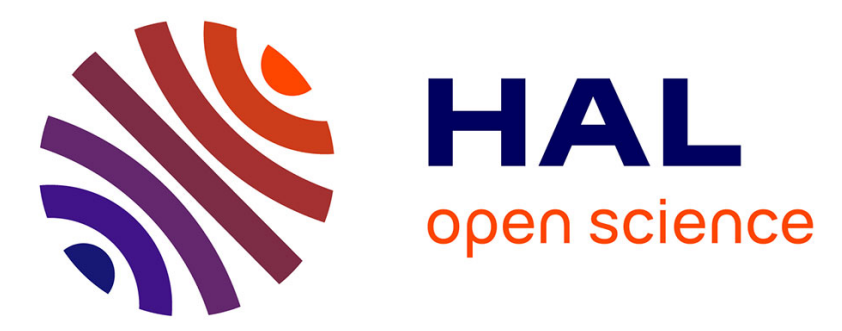

\title{
Melting of 1-Alcohol Mololayers at the Air-Water Interface. I. X-Ray Reflectivity Investigations
}

J. Rieu, J. Legrand, A. Renault, B. Berge, B. Ocko, X. Wu, M. Deutsch

\section{To cite this version:}

J. Rieu, J. Legrand, A. Renault, B. Berge, B. Ocko, et al.. Melting of 1-Alcohol Mololayers at the AirWater Interface. I. X-Ray Reflectivity Investigations. Journal de Physique II, 1995, 5 (4), pp.607-619. 10.1051/jp2:1995152 . jpa-00248183

\section{HAL Id: jpa-00248183 https://hal.science/jpa-00248183}

Submitted on 1 Jan 1995

HAL is a multi-disciplinary open access archive for the deposit and dissemination of scientific research documents, whether they are published or not. The documents may come from teaching and research institutions in France or abroad, or from public or private research centers.
L'archive ouverte pluridisciplinaire HAL, est destinée au dépôt et à la diffusion de documents scientifiques de niveau recherche, publiés ou non, émanant des établissements d'enseignement et de recherche français ou étrangers, des laboratoires publics ou privés. 
Classification

Physics Abstracts

$68.00-68.42-61.10$

\title{
Melting of 1-Alcohol Monolayers at the Air-Water Interface. I. X-Ray Reflectivity Investigations
}

\author{
J.P. Rieu ( $\left(^{1}\right)$, J.F. Legrand $\left({ }^{1}\right)$, A. Renault $\left({ }^{1}\right)$, B. Berge $\left({ }^{1}\right)$, B.M. Ocko $\left({ }^{2}\right)$, \\ X.Z. Wu $\left({ }^{3}\right)$ and M. Deutsch $\left({ }^{4}\right)$ \\ ( ${ }^{1}$ ) Laboratoire de Spectrométrie Physique, U.J.F., B.P.87, 38402 Saint-Martin d'Hères Cedex, \\ France \\ ( $\left.{ }^{2}\right)$ Physics Department, Brookhaven National Laboratory, Upton, NY 11973, U.S.A. \\ $\left({ }^{3}\right)$ Corporate Research Science Laboratory, Exxon Research and Engineering Company, An- \\ nandale, NJ 08801, U.S.A. \\ ( ${ }^{4}$ ) Physics Department, Bar Ilan University, Ramat Gan 52900, Israel
}

(Received 21 October 1994, revised in final form 27 December 1994, accepted 4 January 1995)

Résumé. - La fusion de monocouches de 1-alcool adsorbées à l'interface air-eau est étudiée par réflectivité des rayons $\mathrm{X}$. Cette technique permet une détermination précise de l'épaisseur et de la densité de la monocouche. Les mesures de réflectivité effectuées dans chacune des directions spéculaire et non spéculaire, ont été interprétées à l'aide d'un modèle tenant compte de la résolution expérimentale et de la diffusion par les fluctuations capillaires thermiques. La densité et l'épaisseur de la couche aliphatique présentent une claire discontinuité à la transition solide-liquide. D'importants changements de la compacité de la monocouche sont observés dans chacune des phases solide et liquide sous l'effet de la température. Dans la phase cristalline, les épaisseurs de la monocouche obtenues sont plus petites que celles de molécules dans une conformation "trans-trans". Dans la phase liquide, la valeur de la densité reste $10 \%$ plus élevée que celle de la phase liquide volumique 3D.

\begin{abstract}
The melting of 1-alcohol monolayers adsorbed at the air-water interface has been studied using X-ray reflectivity. This technique allows for an accurate determination of the thickness and the density of the monolayer. The reflectivity, both in specular and non-specular directions, over a large temperature range of different chain lengths (from $\mathrm{C}_{10}$ to $\mathrm{C}_{16}$ ) have been interpreted using a model taking into account the scattering from thermally induced capillarywave fluctuations and the instrumental resolution. The density and the thickness of the aliphatic layer exhibit a clear discontinuity at the solid-liquid transition. Large changes with temperature in the packing of the monolayer are observed in both the solid and the liquid phase. In the crystal phase, the fitted thicknesses are lower than all-trans molecular chain lengths. In the liquid phase, the density remains $10 \%$ larger than in bulk liquid phases.
\end{abstract}




\section{Introduction}

It has been shown recently that fatty alcohols with relatively short chains (from 1-nonanol to 1-hexadecanol) self-assemble in a monomolecular layer at the water surface [1]. In the presence of an excess drop of the insoluble alcohol at the edge of the trough, the amphiphilic monolayer is stable and exhibits a two-dimensional solid-liquid phase transition without detectable thermal hysteresis, although with clear first-order characteristics (e.g., abrupt discontinuities in the ellipsometric angle and in the slope of surface tension). The melting point of the monolayer is about $15 \mathrm{~K}$ above that of the bulk drop. X-ray diffraction studies under grazing incidence have been performed at LURE, Orsay and at ESRF, Grenoble [2,3]. They provide information on the structure of the $2 \mathrm{D}$ crystal which appears as a hexagonal rotator phase with vertical molecules on average but with possible instantaneous tilts of a few degrees.

A thermodynamical analysis of surface tension measurements has allowed us to extract the entropy differences between the drop and the monolayer in the solid and in the liquid phases [4]. These results indicate that the chains are more disordered in the $2 \mathrm{D}$ solid than in the $3 \mathrm{D}$ hexagonal solid. In contrast, the chains are more ordered in the $2 \mathrm{D}$ liquid than in the 3D liquid. Furthermore, thermodynamics predicts the increase of the area per molecule at the melting temperature.

To fully characterize the structure and to account for the entropy difference between $2 \mathrm{D}$ and 3D systems, in-plane structural data from grazing incidence diffraction are not sufficient. Complementary X-Ray reflectivity measurements (specular and non-specular) are required, in particular in the liquid phase, to obtain information about the vertical density profiles.

The reflectivity technique consists of measuring the intensity reflected by an interface as a function of wave vector transfer $q_{z}$ and $q_{y}$ (see the geometry in Fig. 1). Two different measurements have been carried out : (i) specular reflectivity at $q_{y}=0$ as a function of $q_{z}$ and (ii) transverse reflectivity at fixed $q_{z}$ as a function of $q_{y}$. After a presentation of the experimental set-up and the measurements, we shall discuss the model used to fit the data. It takes into account the diffuse scattering and the experimental resolution. The second section is devoted to a description of the fitting procedure. Finally, we discuss the results obtained for the 1-alcohols of different chain lengths.

\section{Experimental Results}

$\mathrm{X}$-rays measurements were carried out on beam line X22B at the National Synchrotron Light Source at Brookhaven National Laboratory (BNL) with $k_{0}=2 \pi / \lambda=4.058 \AA^{-1}$ using the Harward/BNL liquid spectrometer [5]. The beam was projected vertically downward by tilting a $\mathrm{Ge}(111)$ crystal. The incident slits were set so that the entire incident beam impinges on the surface and these settings were always smaller than those of the detector. Thus, the spectrometer resolution was primarily determined by the detector slits (1-2 $\mathrm{mm}$ high, and 4 $\mathrm{mm}$ wide) which were located $600 \mathrm{~mm}$ from the sample.

Samples were obtained from Aldrich SA and used without further purification. We have measured the specular reflectivity curves for monolayers of different alcohols and at different temperatures (both in the $2 \mathrm{D}$ liquid and $2 \mathrm{D}$ solid phases). The compounds studied were 1-decanol $\mathrm{C}_{10} \mathrm{H}_{21} \mathrm{OH}$, 1-undecanol $\mathrm{C}_{11} \mathrm{H}_{23} \mathrm{OH}$, 1-dodecanol $\mathrm{C}_{12} \mathrm{H}_{25} \mathrm{OH}$, 1-tetradecanol $\mathrm{C}_{14} \mathrm{H}_{29} \mathrm{OH}$ and 1-hexadecanol $\mathrm{C}_{16} \mathrm{H}_{33} \mathrm{OH}$. In the following, we will denote the $n$-carbon alcohol by $\mathrm{C}_{n}$. We have also measured the transverse reflectivity for $\mathrm{C}_{11}$ and $\mathrm{C}_{16}$.

Typical normalized specular reflectivity scans are shown in Figure 2 for $\mathrm{C}_{14}$ on water. The data have been divided by Fresnel reflectivity of water, $R_{\mathrm{f}}\left(q_{z}\right) \approx\left(q_{\mathrm{c}} / 2 q_{z}\right)^{4}$ for $q_{z} \gg q_{\mathrm{c}}$ where $q_{\mathrm{c}}=0.0217 \AA^{-1}$ is the critical wavevector for water [5]. The figure displays two measurements 


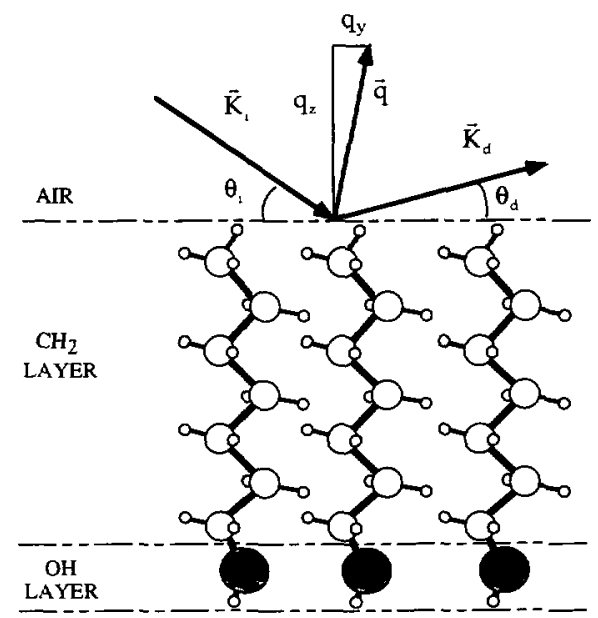

WATER

Fig. 1 - Geometry of the X-ray reflection experiments with $\theta_{2}$ the angle of incidence, $\theta_{d}$ the angle of reflection and $\mathbf{q}$ the wave-vector transfer and schematic view of the packing arrangement taken as a model of the crystalline state of a 1-alcohol monolayer.

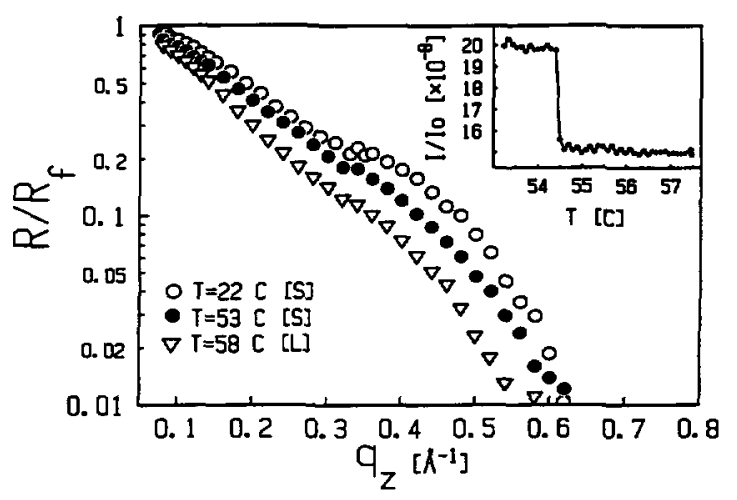

Fig. 2. - Specular reflectivity results (normalized to Fresnel reflectivity of water) for the $\mathrm{C}_{14}$ monolayer in the solid phase at $T=22{ }^{\circ} \mathrm{C}$ and $T=53{ }^{\circ} \mathrm{C}$ and in the liquid phase at $T=58{ }^{\circ} \mathrm{C}$. The inset shows the reflected intensity normalized to the incident beam at $q_{z}=0.22 \AA^{-1}$ as a function of temperature for $\mathrm{C}_{14}$ around melting point $\left(T=5425^{\circ} \mathrm{C}\right)$.

in the solid phase (S) and one in the liquid phase (L). The inset shows the temperature dependence at fixed $q_{z}$, where the rapid change in the intensity at $T=54.5^{\circ} \mathrm{C}$ demonstrates the discontinuous nature of the monolayer melting transition. The prominent oscillations observed in fatty acid monolayers on water [6], which are due to interference between the waves reflected from the air-monolayer and monolayer-water interfaces, are much reduced here. The reduction can be explained by several unfavourable factors:

- The electron density of $-\mathrm{OH}$ polar heads is lower than that of fatty-acids (COOH) and much closer to that of water. This reduces the X-ray optical density contrast at the monolayer water interface. 
- The surface tension of this system (between 25 and $32 \mathrm{mN} / \mathrm{m}$ for all the compounds and temperatures studied [1]) is much lower than that of the water-fatty acid system $(\approx 50 \mathrm{mN} / \mathrm{m})$. Thus, the capillary waves dominated surface roughness [7] is considerably larger. This, and the fairly large period of the oscillations due to the short molecular length (between 10 and $20 \AA$ ) tend to reduce the amplitude of the modulations in the curves.

\section{Model for Surface Scattering of X-Rays}

The reflectivity from a liquid surface is calculated by integrating over the thermally excited capillary mode spectrum with a long wavelength cut-off determined by the spectrometer resolution and a short wavelength cut-off resulting from either the molecular size $[5,7,8]$ or the bending rigidity $K[6,9,11]$. In the present paper, we will formulate the short wavelength cut-off in terms of bending rigidity. From these models, it is predicted that the measured reflectivity deviates from the Fresnel form by a diffuse scattering term and by an effective Debye-Waller-like factor $\exp \left[-\left(\sigma_{\text {eff }} q_{z}\right)^{2}\right]$ with an effective roughness $\sigma_{\text {eff }}$ depending on $q_{z}$. In liquid systems having low surface tensions and consequently significant diffuse scattering, it is necessary to explicitly account for the $q_{z}$ dependence of this roughness [9].

Diffuse scattering arises from the irregular distribution of the heights $z(x, y)$ of the interface. This distribution is characterized by the height-height correlation function $\langle z(x, y) z(0,0)\rangle$ and the mean square amplitude of the oscillations $\left\langle z^{2}\right\rangle$. For a monolayer on water, $\left\langle z(x, y) z(0,0)>\right.$ and $\left\langle z^{2}>\right.$ depend on the gravity constant $g$, the surface tension $\gamma$, and the bending rigidity $K$.

The model used for analysing the reflectivity data assumes that the monolayer is divided into two optically distinct layers: one for the alkyl groups $\mathrm{CH}_{2}$, and one for the $\mathrm{OH}$ head group. We also assume that the fluctuations of heights of the three interfaces are fully correlated so that $\langle z(x, y) z(0,0)\rangle$ and $\left\langle z^{2}>\right.$ are unique. In the analysis which follows we use the Born approximation [5-11].

The general method to calculate the reflected intensity in taking into account instrumental resolution is described in reference [9]. This method has been applied to the case of a monolayer on water in references $[6,11]$. It gives for the specular direction $\left(q_{x}=q_{y}=0\right)$ :

$$
R\left(q_{z}\right)=R_{\mathrm{F}}\left(q_{z}\right) \cdot \exp -\sigma_{\mathrm{eff}}^{2} q_{z}^{2} \cdot \frac{\Gamma\left(\frac{1-\beta q_{z}^{2}}{2} ; \frac{\Delta q_{y}^{2} K}{2 \gamma}\right)}{\sqrt{\pi}} \cdot F\left(\left\{\rho_{\imath}, z_{2}\right\}\right)
$$

with

$$
\beta=\frac{k_{\mathrm{B}} T}{2 \pi \gamma}
$$

The function

$$
F\left(\left\{\rho_{\imath}, z_{\imath}\right\}\right)=\sum_{\imath, \jmath=0}^{2}\left(\rho_{\imath+1}-\rho_{\imath}\right)\left(\rho_{\jmath+1}-\rho_{\imath}\right) \cos \left(q_{z}\left(z_{\imath}-z_{\jmath}\right)\right)
$$

is the interference pattern for two layers with electron densities $\rho_{1}$ and $\rho_{2}$ normalized to that of water $\left(\rho_{0}\right)$, the normalized air density $\rho_{3}$ is assumed zero, $z_{\imath}$ are the mean vertical positions of the different interfaces. The effective roughness is expressed by:

$$
\sigma_{\mathrm{eff}}^{2}=\sigma_{0}^{2}+\sigma_{\mathrm{cw}}^{2}=\sigma_{0}^{2}+\beta \cdot \log \left(\frac{\mathrm{e}^{05772}}{\Delta q_{y}} \sqrt{\frac{\gamma}{2 K}}\right)
$$


where $\sigma_{0}$ and $\sigma_{\text {cw }}$ are the intrinsic and capillary contributions to the surface width [5-10]. A Gaussian shape is assumed for the resolution with a half-width at half-maximum: $\sqrt{2 \ln 2} \Delta q_{y}$ and with a resolution width $\Delta q_{y}$ given by the acceptance of the detector slit height $h$, and its distance from the sample $L$ :

$$
\Delta q_{y}=q_{z} \frac{\Delta \theta_{d}}{2}=\frac{q_{z} \cdot h}{4 \cdot L}
$$

It is important to note that the effective roughness $\sigma_{\text {eff }}$ depends on $q_{z}$ through the instrumental resolution $\Delta q_{y}$. The temperature dependant values of the surface tension $\gamma$ are taken from earlier laboratory measurements [1]. Thus, $\sigma_{0}$ and $K$ are the only parameters in the analysis of the roughness. We will discuss below the influence of the value taken for the intrinsic roughness $\sigma_{0}$ in the evaluation of the bending rigidity $K$.

Finally, $\Gamma(a, b)=\int_{b}^{\infty} t^{a-1} \mathrm{e}^{-t} \mathrm{~d} t$ is the incomplete gamma function [12] and $\Gamma / \sqrt{\pi}$ is the ratio of the total measured intensity (including diffuse scattering) to the true specular intensity. For small $q_{z}$, it is close to unity but for $q_{z}>0.5 \AA^{-1}$ the diffuse contribution becomes dominant. In reference [8], the incomplete gamma function is replaced by a simple gamma function $\Gamma(a)=$ $\int_{0}^{\infty} t^{a-1} \mathrm{e}^{-t} \mathrm{~d} t$ because the short wavelength cut-off (here, $\sqrt{K / \gamma}$ for a monolayer) has been approximated by zero. In our case this approximation is valid up to $q_{z} \approx 0.5 \AA^{-1}$ and we may calculate an explicit expression for the non-specular reflectivity at $q_{x}=0$ :

$$
R\left(q_{y}, q_{z}\right)=\left(\frac{q_{\mathrm{c}}^{4}}{32 q_{z}^{3} k_{0} \sin \theta_{2}}\right) \cdot \exp -\sigma_{\mathrm{eff}}^{2} q_{z}^{2} \cdot \frac{\Gamma\left(\frac{1-\beta q_{z}^{2}}{2}\right)}{\sqrt{\pi}} \cdot F\left(\left\{\rho_{z}, z_{\imath}\right\}\right) \cdot_{1} F_{1}\left(\frac{1-\beta q_{z}^{2}}{2} ; \frac{1}{2} ; \frac{-q_{y}^{2}}{2 \Delta q_{y}^{2}}\right)
$$

where ${ }_{1} F_{1}$ is the Kummer function [12].

2.1. Analysis of Non-Specular Reflectivity. - Let us start by the analysis of the non-specular reflectivity scans as it constitutes only the minor part of this paper. We have measured the rocking scans for the $C_{11}$ and the $C_{16}$. In Figure 3 are shown the $C_{16}$ curves. For small angles of incidence, a sample-rocking scan where the $\operatorname{sum} \theta_{\imath}+\theta_{d}$ is kept constant, is practically identical with a transverse $R\left(q_{y}\right)$ scan at constant $q_{z}$ and $q_{x}=0$. According to equation (5), the entire $q_{y}$ dependence is in the Kummer function except the variation of the intensity due to the varying illuminated area $\left(\operatorname{term} 1 / \sin \theta_{\imath}\right.$ in Eq. (5)). To account for a constant background, transverse scans were performed along $q_{x}$ at $q_{y}=0$. However, the resulting intensity, after substracting this constant background and accounting for the area correction, did not appear symmetrical with respect to $q_{y}$. Since symmetry is required, an adjustable sloping backround parameter was used. Thus, the transverse scans were fitted to equation (5) with three free parameters (i) the slope of the backround line, (ii) the spectrometer resolution $\Delta q_{y}$ and (iii) a normalization constant depending on all other unknown parameters (density, thickness, rigidity) and on $q_{z}$. Good agreement is obtained with $\Delta q_{y}=3.2 \times 10^{-4} q_{z}$ for $\mathrm{C}_{16}$ (and $\Delta q_{y}=3.5 \times 10^{-4} q_{z}$ for $\mathrm{C}_{11}$ ). Using equation (4) with $h=1 \mathrm{~mm}$ and $L=600 \mathrm{~mm}$, the expected value is $4 \times 10^{-4} q_{z}$. The small difference between this value and the fitted ones may be due to the fact that we have used a Gaussian resolution function instead of a more realistic trapezoidal function. In specular scans, the acceptance of the spectrometer was fixed at twice the previous value $(h=2 \mathrm{~mm})$. For these scans we use therefore $\Delta q_{y}=8 \times 10^{-4} q_{z}$.

One observes that the fit with the Kummer function gives a good representation of the diffuse scattering in both the central part $\left(q_{y} / \Delta q_{y}<1\right)$, where it is dominated by the effect of 


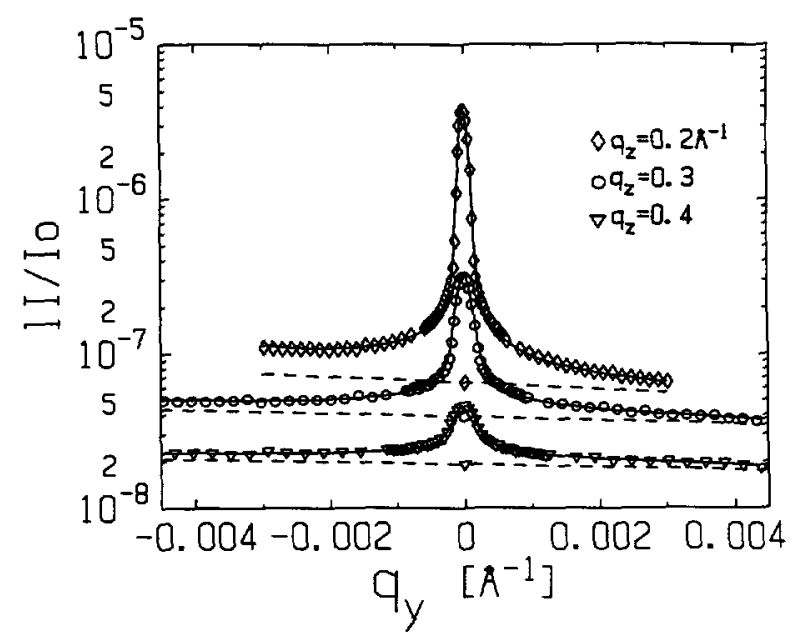

Fig. 3 - Logarithmic plot of the absolute scattered intensity (normalized to the incident beam) vs. $q_{y}$ at severeral values of $q_{z}$ for the $\mathrm{C}_{16}$ at $273^{\circ} \mathrm{C}$ (where $\gamma=25 \mathrm{mN} / \mathrm{m}$ ) The solid curves represents the best fit with the theoretical expression (Eq. (5)) added to the backgrounds (with fitted slopes shown as dashed lines and with central $q_{y}=0$ measured points) as described in the text.

resolution and in the asymptotic regions $\left(q_{y} / \Delta q_{y} \gg 1\right)$ where it follows the power-law behaviour $q_{y}^{\left(\beta q_{z}^{2}-1\right)}$. This gives confidence in the description employing the capillary-wave formalism for the present measurements.

2.2. Fitting Procedure for Specular Reflectivity. - Let us return now to the analysis of the specular reflectivity scans. The data are fitted using equation (1) with three or four parameters. Rigorously, it would be necessary to use six parameters: the bending rigidity $K$, the intrinsic roughness $\sigma_{0}$, two densities and two thicknesses for the aliphatic layer $\left(\mathrm{CH}_{2}\right)$ and head-group layer (OH) (Fig. 1). Unfortunately, as mentioned above, our data do not show prominent structure into which the fitting procedure can find a well-defined minimum. It is therefore important to reduce the number of free parameters in the fit as much as possible. The strong correlation between $K$ and $\sigma_{0}$ does not allow simultaneous variation of both parameters. Since the choice of $\sigma_{0}$ only affects $K$, we have set $\sigma_{0}=1.1 \AA$ (the value obtained in Ref. [7] for the liquid alkanes) for much of the analysis. We also make the assumption that the head group layer does not contain any water molecules or alkyl group, and that the aliphatic tail layer contains all the $n \mathrm{CH}_{2}$ groups. Such a hypothesis seems reasonable for the solid phase (Fig. 1). Indeed, the terminal C-OH bond is generally supposed to sit almost perpendicular to the water surface [13]. This clearly differentiates the two layers. Moreover, the areas per molecule are sufficiently small $\left(\mathrm{A}<21.5 \AA^{2}\right)$ to prevent water penetration into the $\mathrm{OH}$ layer.

Thus, from the two parameters of the aliphatic tail layer, namely the electronic density or equivalently the mean volume per $\mathrm{CH}_{2}, V_{2}$, and the tail thickness $L_{2}$, we get the mean area per molecule $A=n V_{2} / L_{2}$. Then, the head region is characterized by only one parameter, for instance its thickness $L_{1}$, as $V_{1}=L_{1} A$. It is possible to reduce the number of free parameters even further by constraining the area per molecule to that obtained by X-rays diffraction for temperatures in the solid phase, or from thermodynamics calculations [4] for temperatures close to the melting point. For each fit, the parameters are adjusted to minimize 
a)

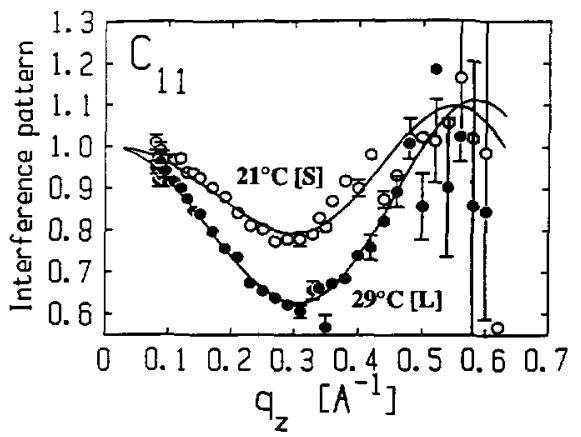

b)

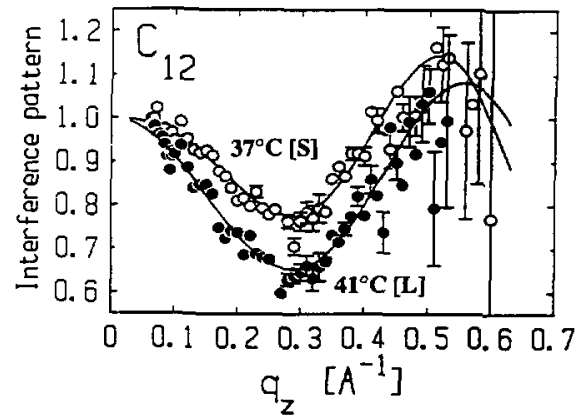

c)

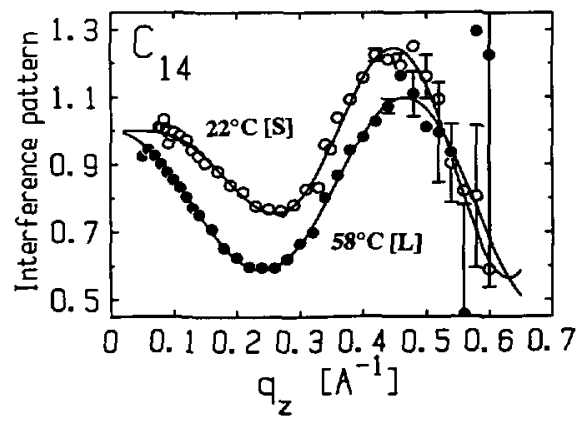

Fıg. 4. - Normalized reflectivity curves $R\left(q_{z}\right) \sqrt{\pi} /\left[R_{\mathrm{F}} q(z) \cdot \exp -\sigma_{\mathrm{eff}}^{2} q_{z}^{2} \cdot \Gamma\left(0.5-05 \beta q_{z}^{2} ; 0.5 \Delta q_{y}^{2} K / \gamma\right)\right]$ showing interference patterns for. $\mathrm{C}_{11}(\mathrm{a}), \mathrm{C}_{12}$ (b) and $\mathrm{C}_{14}$ (c) (values of the fitted parameter $\sigma_{\text {eff }}$ used for the normalization are summarized in Tab. I). The solid lines are the corresponding fits.

$\chi^{2}=\sum_{\imath=1}^{N_{\text {data }}}\left(y_{\text {fit }}-y_{\text {data }}\right)_{\imath}^{2} /\left(N_{\text {data }}-N_{\text {par }}\right) \cdot \delta_{\imath}^{2}$, the standard error deviation (where $\delta_{\imath}$ is the experimental error, $N_{\text {par }}$ the number of free parameters) and the errors bars are estimated from the requirement $\Delta \chi^{2}=\chi^{2}-\chi_{\min }^{2}=1$ in the parameter space.

The results of the fits are shown in Figure 4 for $C_{11}, C_{12}$ and $C_{14}$, and the whole set of fitted parameters is listed in Table I. In order to emphasize the interference term given by equation (2), the data and the fit have been normalized by both the Fresnel Reflectivity and by the fitted capillary wave terms. In contrast to the uncorrected reflectivity shown in Figure 2 , one can now see actual ascillations up to the second maximum around $q_{z}=0.45 \AA^{-1}$ for the $\mathrm{C}_{14}$ (Fig 4c) or around $q_{z}=0.55 \AA^{-1}$ for the $\mathrm{C}_{11}$ (Fig. 4a). For each compound, both the amplitude and the period of the oscillation is slightly shifted from the solid state (S) to the 
Table I. - Parameters extracted from the fits of all the specular reflectivaty measurements. The phases, [S] for solid or [L] for liquid are indicated after the temperature. Double underlined parameters are constrained ones.

\begin{tabular}{|r|r|r|r|r|r|r|r|}
\hline$n$ & $T\left[{ }^{\circ} \mathrm{C}\right]$ & $\begin{array}{c}V^{2}\left[\AA^{3}\right] \\
( \pm 0.3)\end{array}$ & $\begin{array}{c}L^{2}[\AA] \\
( \pm 1)\end{array}$ & $\begin{array}{c}L^{1}[\AA] \\
( \pm 0.1)\end{array}$ & $\begin{array}{r}A\left[\AA^{2}\right] \\
( \pm 1.5)\end{array}$ & $\begin{array}{c}\sigma_{\text {eff }}[\AA] \\
( \pm 0.1)\end{array}$ & $\chi^{2}$ \\
\hline \hline 10 & $9.5[\mathrm{~S}]$ & 25.0 & $\underline{\underline{11.9}}$ & 1.10 & $\underline{\underline{21.3}}$ & 4.20 & 33.2 \\
& & 24.7 & 13.3 & 1.20 & 18.5 & 422 & 326 \\
\hline 10 & $13.5[\mathrm{~S}]$ & 25.2 & $\underline{\underline{11.7}}$ & 1.05 & $\underline{\underline{21.5}}$ & 4.19 & 1.8 \\
& & 25.0 & 12.2 & 1.10 & 20.6 & 4.22 & 1.7 \\
\hline 10 & $14.5[\mathrm{~L}]$ & 25.9 & $\underline{\underline{11.0}}$ & 1.00 & $\underline{\underline{23.5}}$ & 428 & 6.5 \\
& & 25.1 & 13.4 & 1.20 & 18.7 & 4.43 & 3.5 \\
\hline 10 & $21.7[\mathrm{~L}]$ & 26.5 & 11.2 & 1.00 & 23.7 & 4.23 & 2.6 \\
\hline \hline 11 & $21.0[\mathrm{~S}]$ & 25.3 & $\underline{13.1}$ & 1.15 & $\underline{\underline{21.3}}$ & 4.11 & 2.8 \\
& & 25.2 & 13.9 & 1.20 & 20.2 & 4.17 & 2.3 \\
\hline 11 & $29.0[\mathrm{~L}]$ & 26.7 & $\underline{\underline{12.0}}$ & 0.95 & $\underline{\underline{24.5}}$ & 4.24 & 1.5 \\
& & 26.3 & 13.1 & 1.00 & 22.2 & 4.31 & 1.3 \\
\hline \hline 12 & $32.0[\mathrm{~S}]$ & 25.2 & $\underline{\underline{14.3}}$ & 1.10 & $\underline{\underline{21.1}}$ & 431 & 10.9 \\
& & 25.0 & 15.5 & 115 & 19.3 & 437 & 9.5 \\
\hline 12 & $37.5[\mathrm{~S}]$ & 25.4 & $\underline{\underline{14.2}}$ & 1.10 & $\underline{\underline{21.4}}$ & 4.28 & 3.9 \\
& & 25.3 & 15.0 & 1.15 & 20.2 & 4.31 & 3.8 \\
\hline 12 & $41.5[\mathrm{~L}]$ & 26.6 & $\underline{\underline{12.5}}$ & 0.90 & $\underline{\underline{25.5}}$ & 4.43 & 4.9 \\
& & 26.0 & 14.3 & 1.00 & 21.9 & 4.50 & 4.3 \\
\hline 12 & $56.5[\mathrm{~L}]$ & 27.2 & 10.3 & $\underline{\underline{0.00}}$ & 31.6 & 4.55 & 11 \\
& & 25.9 & 14.8 & 1.00 & 20.9 & 4.77 & 98 \\
\hline \hline 14 & $22.0[\mathrm{~S}]$ & 25.0 & $\underline{\underline{173}}$ & 1.00 & $\underline{\underline{20.3}}$ & 3.98 & 4.1 \\
& & 25.2 & 16.6 & 0.95 & 21.2 & 393 & 2.8 \\
\hline 14 & $53.5[\mathrm{~S}]$ & 25.5 & $\underline{\underline{16.7}}$ & 1.00 & $\underline{\underline{21.5}}$ & 415 & 7.7 \\
& & 25.7 & 16.0 & 1.00 & 22.6 & 4.11 & 5.5 \\
\hline 14 & $58.5[\mathrm{~L}]$ & 27.3 & $\underline{\underline{13.9}}$ & 1.00 & $\underline{\underline{27.5}}$ & 437 & 4 \\
& & 27.1 & 15.0 & 0.85 & 25.3 & 4.42 & 3.4 \\
\hline 14 & $68.5[\mathrm{~L}]$ & 27.6 & 12.7 & 0.00 & 30.4 & 4.39 & 2.2 \\
\hline \hline 16 & $27.0[\mathrm{~S}]$ & 24.8 & $\underline{\underline{19.8}}$ & 1.00 & $\underline{\underline{20.0}}$ & 4.30 & 13 \\
& & 25.1 & 18.0 & 0.90 & 22.3 & 4.20 & 1.9 \\
\hline
\end{tabular}

liquid state (L) of the monolayer.

The best fits correspond to a $\chi_{\mathrm{m} n}^{2}$ of about 1.5 , i.e. close to the experimental error. For $\mathrm{C}_{16}$, the fit obtained with the area constrained at $20 \AA^{2}$ has a large $\chi_{\min }^{2}$ while a reasonable fit is obtained when the area is allowed to vary up to a value of $22.3 \AA^{2}$. We suspect that this discrepancy is due in part to fortuitous lack of equilibrium between the drop and the monolayer. Indeed, the $\mathrm{C}_{16}$ was measured only at a single low temperature, ten degrees below the melting 
of the excess drop. Allowing the area to float for the other chain molecules has a small effect on $\chi_{\min }^{2}$. For instance, for $\mathrm{C}_{14}$ the $\chi_{\min }^{2}$ with the area fixed is only $30 \%$ higher than the fit with the floating area. The small discrepancy in tail length (the more sensitive parameter) between the two fits may be due to the simplicity of the previous assumption regarding the layer composition. In Figure $4 \mathrm{c}$, we show the normalized reflectivity data for $\mathrm{C}_{14}$ at $22^{\circ} \mathrm{C}$ and $58^{\circ} \mathrm{C}$. The four parameter fits, shown as a solid line, provide an excellent description of the reflectivity.

Beyond $q_{z}=0.5 \AA^{-1}$, the experimental points are dispersed due to poor statistics (specular reflectivity $\left.<10^{-8}\right)$. For the shortest chains $\left(\mathrm{C}_{10}, \mathrm{C}_{11}, \mathrm{C}_{12}\right)$, the second maximum is not clearly evident. The acuracy in the fitted parameters is much lower and the fits without the area constraint often give non physical parameter values. For instance the thickness for $\mathrm{C}_{10}$ at $9.5^{\circ} \mathrm{C}$ or for $\mathrm{C}_{12}$ at $37.5^{\circ} \mathrm{C}$ are much larger than the tail lengths in the all-trans conformation $L_{2}^{0}=n \times 1.25 \AA\left(L_{2}^{0}=12.5 \AA\right.$ for $\mathrm{C}_{10}$ and $15 \AA$ for $\left.\mathrm{C}_{12}\right)$. So for short chains, we systematically choose the results obtained with the area constraint. Figures $4 \mathrm{a}$, b show the fitted data of $\mathrm{C}_{11}$ and $\mathrm{C}_{12}$.

\section{Results}

We have displayed the results listed in Table $I$ for the mean volume $V_{2}$, the thickness of the aliphatic layer $L_{2}$ and the bending rigidity $K$ in Figures 5,6 and 7, respectively. The uncertainties for $V_{2}, L_{2}$ and $L_{1}$ are $\pm 0.3 \AA^{3}, \pm 1 \AA$ and $\pm 0.1 \AA$, respectively. The very

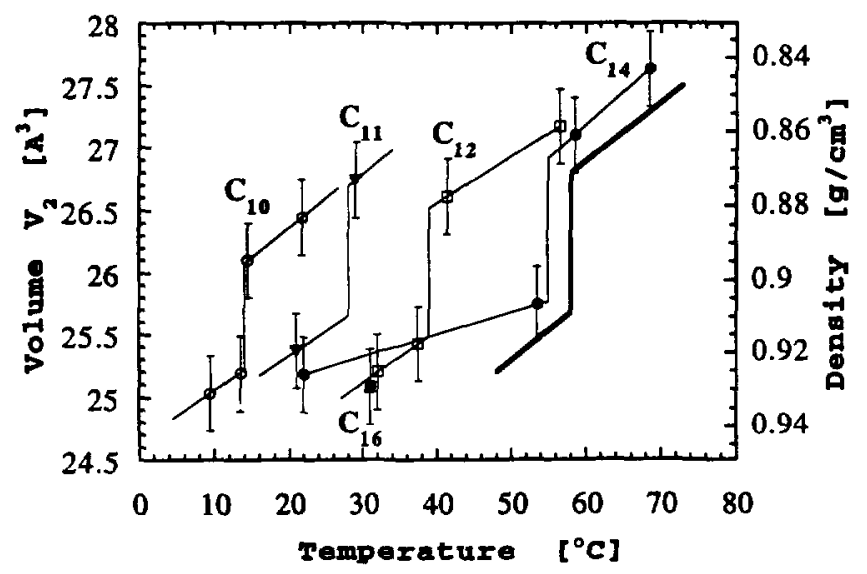

Fig. 5. - Mean volume per $\mathrm{CH}_{2}$ group $\left(V_{2}\right)$ and corresponding density as a function of temperature for $\mathrm{C}_{10}, \mathrm{C}_{11}, \mathrm{C}_{12}, \mathrm{C}_{14}, \mathrm{C}_{16}$. The solid line is a guide for eyes The thick line is a measurement in a 14-carbon lecithin bilayer by dilatometry [16].

satisfactory accuracy for $V_{2}$ is due to the fact that the $-\mathrm{OH}$ heads layer is very thin and has little effect on the first part of the reflectivity curve $\left(q_{z}<0.25 \AA^{-1}\right)$.

As shown in the inset in Figure 2, the reflectivity at a fixed $q_{z}$ presents a clear discontinuity exactly at the solid-liquid transition temperature. From the results of the fit at several fixed temperatures, the mean volume per $\mathrm{CH}_{2}$ (Fig. 5) also exhibits a discontinuity at the transition temperature. The volume change at the melting is only $5 \%$ compared with an approximate 
$12 \%$ change in the $3 \mathrm{D}$ melting transition of hydrocarbons [14]. In the solid phase, the values obtained for $V_{2}$ (between 25 and $25.75 \AA^{3}$ ) are comparable to those measured in the rotator phase of alkanes, in 3D systems, and in the surface crystalline monolayer of liquid alkanes [15]. Values of the mean volume per $\mathrm{CH}_{2}$ in the $2 \mathrm{D}$ liquid phase (between 26 and $27.6 \AA^{3}$ ) are two or three $\AA^{3}$ less than in the $3 \mathrm{D}$ liquid phases of all aliphatic chains.

We also show in Figure 5 the only dilatometric measurement published to date for a similar 2D system, to the best of our knowledge. These are measurements by Nagle and Wilkinson [16] for $\mathrm{C}_{14}$ lecithin bilayers. The curve corresponds also to a $2 \mathrm{D}$ hexagonal-crystalline to liquid transition, as elucidated by the authors. The temperature scale has been shifted in order to be next to tetradecanol fitted curve. The similarity between the behaviour of alcohols and lecithins is striking.

In Figure 6, one can see that the thickness of the $\mathrm{CH}_{2}$ layer decreases with temperature for each compound studied. The discontinuity at the transition temperature is not as evident as it is for the mean volume per $\mathrm{CH}_{2}$ because it is of same order of magnitude as the error bars, but it still exasts. At the lowest temperatures, the thicknesses of the layers $\mathrm{L}_{2}$ are smaller than thoses of vertical all-trans chains $L_{2}^{0}$ (for $\mathrm{C}_{12}, L_{2}=14.5 \AA, L_{2}^{0}=15 \AA$ and for $\mathrm{C}_{14}$, $L_{2}=16.6 \AA, L_{2}^{0}=17.5 \AA$ ).

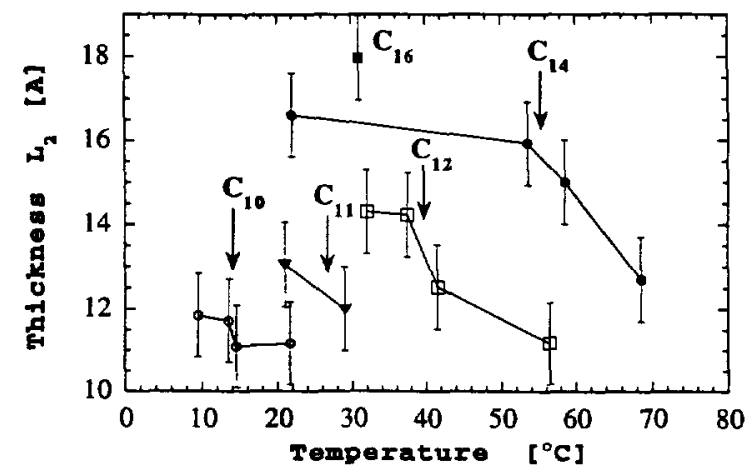

Fig. 6. - Thickness of the alkyl layer as a function of temperature Arrows indicate the 2D melting temperatures

Finally, Figure 7 presents our results for the bending rigidity $K$ using for the intrinsic width $\sigma_{0}=1.1 \AA$. The error bars are of order $80 \% ; 40 \%$ comes from the $0.1 \AA$ uncertainty in the fitted $\sigma_{\text {eff }}$, and $40 \%$ comes from a $1 \mathrm{mN} / \mathrm{m}$ uncertainty in the surface tension. It is important to note that the choice of $\sigma_{0}$ drastically influences the absolute value of $K$ but not the quality of the fit. Indeed, if $\sigma_{0}=0.7 \AA$ is taken, a value which satisfactorily accounts for the minimum effective bending rigidity of a liquid surface $\left(K \approx 0.12 k_{\mathrm{B}} T\right)$ due to a coupling between modes [17], then $K$ is found approximatively two times lower than in Figure 7. Nevertheless, both choices give comparable relative changes in the bending rigidity either with chain length or with temperature. In the solid phase, the bending rigidity value is clearly larger than in the liquid phase. For instance, for the $\mathrm{C}_{14}, K \approx 17 k_{\mathrm{B}} T$ at $22^{\circ} \mathrm{C}$ (solid) and $0.3 k_{\mathrm{B}} T$ at $68.5^{\circ} \mathrm{C}$ (liquid). This gives the short wavelength cut-offs $\sqrt{K / \gamma} \approx 15 \AA$ and $2 \AA$, respectively. Thus, in the solid phase this cut-off is linked to the rigidity, while in the liquid phase it may be related to a molecular dimension as in reference [7]. In so doing, for the solid phase, $K$ increases with chain length, as predicted by simple arguments of flexure elasticity [6]. Even for the longest chains, 


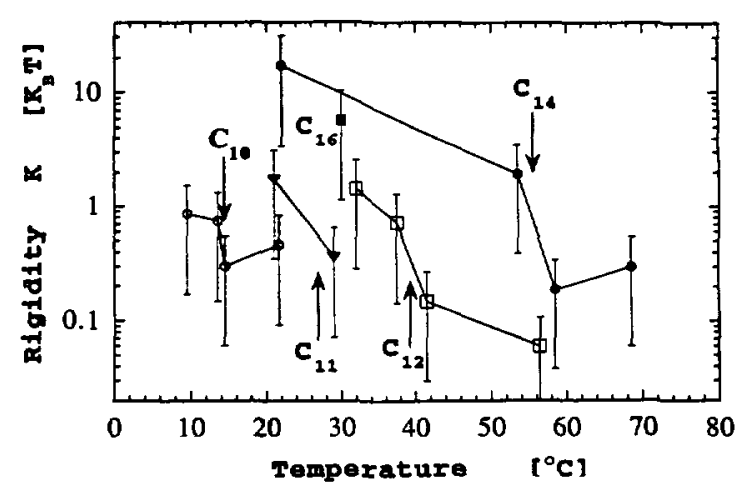

Fig. 7 - Logarithmic plot of the bending rıgıdıty modulus as a function of temperature Arrows indicate the $2 \mathrm{D}$ melting temperatures.

our estimate of $K$ remains one order of magnitude lower than for fatty-acid monolayers. This is attributed to the softness of the rotator phase of alcohol monolayers in comparison with higher density and more ordered crystalline phases of fatty-acids monolayers.

\section{Discussion}

Upon heating above the melting temperature, a continuing decrease in both the density and the thickness $L_{2}$ is observed, in particular for $\mathrm{C}_{12}$ and $\mathrm{C}_{14}$ (Figs. 5, 6). In the solid phase, one observes a comparable evolution with temperature. This indicates large changes in the packing of the $2 \mathrm{D}$ liquid and the $2 \mathrm{D}$ solid with temperature, hence a large heat capacity. It is important to note that these structural features of our alcohol monolayers have been already observed in both the dilatometric measurements of Nagle [16] and in the Molecular Dynamics simulations for $\mathrm{C}_{15}$ monolayers by Karaborni and Toxvaerd [18].

The higher density of the $2 \mathrm{D}$ liquid, as compared to the $3 \mathrm{D}$ liquid, is perhaps the most significant result of this study since this phase was very poorly known. This is not really surprising in view of the orientation and the confinement of the elongated molecules to a common plane. The anchorage of the head group by the water and the Van der Waals interactions can more readily induce smectic-like orientational order along the surface normal, hence a denser in-plane packing than in a 3D liquid. This smectic-like order is consistent with previous thermodynamic studies [4], where we have shown that the entropy per molecule of a liquid monolayer is approximatively $2 k_{\mathrm{B}}$ lower than in the bulk liquid. This difference may be attributed in part to the translational degree of freedom along the vertical which is frozen for the $2 \mathrm{D}$ system $\left(\approx 1 k_{\mathrm{B}}\right)$, but also to the lower conformational disorder of the oriented $2 \mathrm{D}$ liquid chains $\left(\approx 1 k_{\mathrm{B}}\right)$.

The fact that the thickness of the layer in the solid phase is systematically smaller than the length of vertical all-trans chains should be considered with some caution. Firstly, because the uncertainty in the fitted thickness is of the order of $1 \AA$, and secondly because, as mentioned above, the layer model may be oversimplified: the head layer might be penetrated by water molecules or by the first alkyl group. However, $\mathrm{Wu}$ et al. have obtained the same results from reflectivity measurements of the surface crystalline monolayer of liquid alkanes [15], and several independent studies support the conclusion that the reduced thickness as compared to $L_{2}^{0}$ is a real effect. The Bragg rods obtained by grazing incidence diffraction have shown a profile significantly broader than the calculated form factor of an all-trans molecule [3]. The interpretation given was that chains are rotating around the vertical axis with a root mean 
square tilt angle of their principal axis of 5-7 degrees. Obviously, if the chains remain rigid, such a small tilt doesn't affect significantly the layer thickness. However, the Bragg rod broadening can also be attributed to an average chain length decrease. For instance, gauche defects such as GTG' kinks along the alkyl chain or end-gauches [19] would decrease the layer thickness by $1.25 \AA$. Moreover,thermodynamic calculations indicate that the entropy per molecule in the $2 \mathrm{D}$ rotator phase is approximatively $2 k_{\mathrm{B}}$ higher than in the $3 \mathrm{D}$ rotator phase [4]. This suggests that there already exists some conformational disorder in the solid phase, and thus that the thickness of the monolayer is lower than $L_{2}^{0}$. Such a slight conformational disorder is supported by Molecular Dynamics simulations [18]: the authors point to two successive transitions: (1) a continuous transition characterized by a change in chain conformation from all-trans to gauche defected conformation in the solid phase, and (2) a first-order solid-liquid transition at $A=22 \AA^{2}$ which coincides with our hexagonal-to-melt transition at $21.5 \AA^{2}$ observed by grazing incidence diffraction.

\section{Conclusion}

Despite the low contrast in the specular reflectivity curves, the modified capillary wave model presented here provides reasonable estimates of the monolayer thickness and mean volume per $\mathrm{CH}_{2}$. To our knowledge, these are the first results of this type showing temperature and chain length dependences, particularly for the volume per $\mathrm{CH}_{2}$, a quantity very poorly known in $2 \mathrm{D}$ systems such as monolayers. Usually, it is assumed that the aliphatic medium density is constant within the liquid and solid phases of adsorbed monolayers on water. In this paper, we have obtained a volume increase at the melting transition of about $5 \%$, a lower value than in the $3 \mathrm{D}$ melting $(+12 \%)$. This is attributed to the high density of the liquid phase and more generally to the weaker first order melting transition in 2D systems. In addition, the results of fitted thicknesses in the $2 \mathrm{D}$ crystalline rotator phase are smaller than the lengthes of all-trans chains, a trend already observed in grazing incidence diffraction (Bragg rods). This study also provides a crude estimate of the change in the bending rigidity of the monolayer in the solid phase with temperature and chain length.

Further studies over larger temperature ranges and for the larger molecules $\left(\mathrm{C}_{14}, \mathrm{C}_{15}, \mathrm{C}_{16}\right)$ are recommanded to get deeper insight into these structural features and phase transitions.

Forthcoming papers will present complementary results of neutron reflectivity and of grazing incidence diffraction.

\section{Acknowledgments}

We wish to thank J. Daillant, S.K. Sinha and M. Vallade for useful discussions and a critical reading of the manuscript. Brookhaven National Laboratory is supported by thè Division of Materials Research, U.S. Departement of Energy under contract No. DE-AC02-76CH00016. The liquid spectrometer is partially supported through NSF Grant No. NSF-DMR-89-20490. This work was supported in part by the U.S.-Israel Binational Science Foundation, Jerusalem.

\section{References}

[1] Berge B. and Renault A., Europhys. Lett. 21 (1993) 773.

[2] Renault A., Legrand J.F., Goldmann M. and Berge B, J. Phys. II France 3 (1993) 761 
[3] Legrand J.F., Renault A., Konovalov O., Chevigny E., Als-Nielsen J., Grübel G. and Berge B., Thun Sol Fulms 248 (1994) 95.

[4] Berge B., Konovalov O., Lajzerowicz J., Renault A., Rieu J.P., Vallade M., Als-Niesen J., Grübel G. and Legrand J.F, Phys Rev. Lett. 73 (1994) 1652.

[5] Braslau A., Pershan P.S., Swislow G, Ocko B.M. and Als-Nielsen J., Phys. Rev. A 38 (1988) 2457; Schwartz D.K., Schlossman M.L., Kawamoto E.K., Kellog G., Pershan P.S. and Ocko B.M., Phys. Rev. A 41 (1990) 5687.

[6] Daillant J., Bosio L., Harzallah B. and Benattar J J., J. Phys II France 1 (1991) 149.

[7] Ocko B.M , Wu X.Z, Sirota E B., Sinha S.K. and Deutsch M., Phys. Rev. Lett. 72 (1994) 242.

[8] Sanyal M K., Sinha S.K. and Huang K.G. and Ocko B.M, Phys. Rev Lett. 66 (1991) 628.

[9] Daillant J. and Belorguey O., J. Chem. Phys. 97 (1992) 5824, J. Chem Phys. 97 (1992) 5837

[10] Sinha S K., Sirota E.B. and Garoff S., Phys. Rev. B 38 (1988) 2297.

[11] Bourdieu L, Daillant J, Chatenay D., Braslau A. and Colson D., Phys. Rev. Lett. 72 (1994) 1502.

[12] Gradshteyn and Ryzhik, Table of Integrals, Series and Products (Academic Press, New York, 1965).

[13] Jacquemain D., Leveiller F., Weinbach S O., Lahav M., Kjaer K., Leiserowitz L and Als-Nielsen J., J. Am. Chem. Soc. 113 (1991) 7684.

[14] Small D.M., The physical Chemistry of Lipids, Handbook of Lipid Research Vol. 4 (Plenum Press, New York, 1986).

[15] Wu X.Z., Sirota E B., Sınha S.K., Ocko B.M. and Deutsch M, Phys. Rev. Lett. 70 (1993) 958.

[16] Nagle J.F. and Wilkinson D.A , Bıophys. J 23 (1978) 159.

[17] Meunier J., J. Phys. France 48 (1987) 1819.

[18] Karaborni S. and Toxvaerd S , J. Chem. Phys. 96 (1992) 5505.

[19] Maroncelli M., Strauss H.L. and Snyder R.G., J. Chem. Phys. 82 (1985) 2811. 